PrisCila Ribeiro Huguet ${ }^{1}$

SIRLEI SIANI MORAIS ${ }^{2}$

MARIA JOSÉ DUARTE OSIS

Aarão Mendes PInTO-Neto ${ }^{4}$

Maria Salete Costa Gurgel ${ }^{4}$

\section{Qualidade de vida e sexualidade de mulheres tratadas de câncer de mama}

\author{
Quality of life and sexuality of women treated for breast cancer
}

\section{Palavras-chave \\ Qualidade de vida Neoplasias da mama/cirurgia Sexualidade \\ Keywords \\ Quality of life Breast neoplasms/surgery Sexuality}

\section{Resumo}

OBJETIVO: avaliar a qualidade de vida e aspectos da sexualidade de mulheres com câncer de mama segundo o tipo de cirurgia e características sociodemográficas. MÉTODOS: realizou-se um estudo de corte transversal com 110 mulheres traładas há pelo menos um ano por câncer de mama no Centro de Atenção Integral à Saúde da Mulher da UNICAMP. A qualidade de vida foi avaliada por meio do questionário WHOQOL-bref e as questões sobre sexualidade, por um questionário específico, no qual se utilizou o coeficiente alpha de Cronbach para verificar validade e concordância das respostas (alpha=0,72) e a técnica de análise fatorial com critério de autovalor e rotação máxima de variância, resultando em dois componentes assim denominados: intrínseco ou intimidade (como a mulher se vê sexualmente) e extrínseco ou atratividade (como a mulher acredita que os outros a veem sexualmente). As variáveis sociodemográficas foram avaliadas nos domínios do questionário da OMS e nos componentes de sexualidade por meio do teste de Kruskal-Wallis seguido pelo teste de Mann-Whitney e pela correlação de Spearman. RESULTADOS: idade, escolaridade, tipo de cirurgia e tempo desde a cirurgia não influenciaram a qualidade de vida nos domínios físico, meio ambiente, psicológico e relações sociais. Mulheres com relacionamento marital estável tiveram escores maiores nos domínios psíquico $(p=0,04)$ e relações sociais $(p=0,02)$. Maior nível socioeconômico influenciou a qualidade de vida nos domínios físico $(p=0,01)$ e meio ambiente $(p=0,002)$. Em relação à sexualidade, houve influência da idade no componente extrínseco ( $p=0,0158)$. Mulheres com relacionamento marital estável tiveram escores maiores de qualidade de vida em ambos os componentes de sexualidade. Maior escolaridade influenciou positivamente no fator intrínseco. Mulheres submetidas à quadrantectomia ou à mastectomia com reconstrução imediata apresentaram melhores escores em relação à atratividade quando comparadas às mastectomizadas sem reconstrução. CONCLUSÕES: melhor nível socioeconômico e de escolaridade, relação marital estável e cirurgia com conservação mamária estão associados a melhores taxas de qualidade de vida, inclusive a sexual.

\section{Abstract}

PURPOSE: to evaluate the quality of life and sexuality features of women with breast cancer, according to the type of surgery they underwent and their sociodemographic characteristics. METHODS: transversal study with 110 women treated for breast cancer, for at least one year in the Centro de Atenção Integral à Saúde da Mulher of UNICAMP. The quality of life was assessed by the WHOQOL-bref questionnaire, and the issues on sexuality, by a specific questionnaire in which Cronbach's Alpha coefficient was used to validate the concordance of responses (alpha=0.72) and the technique of factor analysis, with the criterion of self value and variance maximum rotation, resulting in two components: intrinsic or intimacy ( how the woman sees herself sexually) and extrinsic or attractiveness (how the woman believes the others see her sexually). Sociodemographic variables have been assessed according to the WHO questionnaire, and the sexuality components, through the Kruskal-Wallis followed by the Mann-Whitney's test and Spearman correlation test. RESULTS: age, schooling, type of surgery and lapse of time from the surgery did not influence the quality of life concerning physical, environmental, and psychological aspects, as well as the social relationships. Women with a stable marital relationship got higher scores in the psychological area $(p=0.04)$ and in the area of social relationships $(p=0.02)$. Higher socioeconomic level influenced the quality of life concerning physical appearance $(p=0.01)$ and environment ( $p=0.002)$. Regarding the sexuality, age had influence in the extrinsic component ( $p=0.0158)$. Women Rua Alexander Flemming, 101 CEP 13083-970 - Campinas/SP Fone/Fax: (19) 3521-9305 E-mail: salete@caism.unicamp.br

Recebido

$3 / 9 / 08$
Ambulatório de Oncologia Mamária do Centro de Atenção Integral à Saúde da Mulher da Universidade Estadual de Campinas UNICAMP - Campinas (SP), Brasil.

'Médica Ginecologista e Mastologista; Mestre em Tocoginecologia pela Universidade Estadual de Campinas - UNICAMP - Campinas (SP), Brasil.

2 Estatística do Centro de Atenção Integral à Saúde da Mulher (CAISM) da Universidade Estadual de Campinas - UNICAMP Campinas (SP), Brasil.

${ }^{3}$ Doutora, Professora do Centro de Pesquisas em Saúde Reprodutiva de Campinas - CEMICAMP - Campinas (SP), Brasil.

4 Livre-docente; Professor-associado do Departamento de Tocoginecologia da Faculdade de Ciências Médicas da Universidade Estadual de Campinas - UNICAMP - Campinas (SP), Brasil. 
with a stable marital relationship had higher scores of quality of life in both components of sexuality. Higher schooling influenced in a positive way the intrinsic factor. Women submitted to quadrantectomy or mastectomy with immediate breast reconstruction showed higher scores relating to attractiveness in comparison to mastectomized women without reconstruction. CONCLUSIONS: better socioeconomic level and better schooling, stable marital relationship and surgery with breast conservation are linked to better rates of quality of life, including sexuality.

\section{Introdução}

Segundo a Cancer Statistics, em 2008 houve 182.460 novos casos de câncer de mama em mulheres nos Estados Unidos, e 40.480 mortes em decorrência dele ${ }^{1}$. De acordo com dados do Instituto Nacional do Câncer, em estimativa feita para 2008, serão diagnosticados no Brasil 49.470 casos novos, sendo a segunda neoplasia mais incidente em mulheres ${ }^{2}$. Com o aumento das taxas de detecção precoce e com a melhoria nos tratamentos oferecidos, sabe-se que aproximadamente $50 \%$ das mulheres sobreviverão por, pelo menos, quinze anos após o diagnóstico ${ }^{1}$ e deverão ajustar-se às sequelas cirúrgicas ${ }^{3}$.

A ablação de um órgão como a mama pode trazer agravos à qualidade de vida, à satisfação sexual e recreativa ou à prática de esportes. Em contrapartida, parece haver satisfação maior nas mulheres submetidas à cirurgia conservadora ${ }^{4-6}$.

Em um primeiro momento, um diagnóstico como o do câncer de mama certamente causa um efeito devastador na vida de uma mulher. O medo de morrer é a questão principal, e a busca pelo tratamento adequado e pela cura é uma constante ${ }^{7}$. Aos poucos e, sobretudo, após a fase aguda do tratamento, há uma tendência a se retornar à qualidade de vida de antes do diagnóstico, ou mesmo a ocorrerem mudanças positivas na qualidade de vida, com oportunidade de crescimento pessoal. O enfrentamento da doença leva, na maioria dos casos, a um maior sentido na vida e à reestruturação de prioridades ${ }^{8,9}$.

Qualidade de vida é termo de difícil definição, pois inclui uma variedade potencial de condições que afetam: a percepção do indivíduo, seus sentimentos e comportamentos relacionados ao seu funcionamento diário, incluindo a sua condição de saúde e intervenções médicas. Essa passou a ser considerada a terceira dimensão a ser estudada em pesquisas, além da eficácia e da segurança ${ }^{10}$. A oncologia é uma das áreas que mais tem avaliado a qualidade de vida, pois os tratamentos, por vezes agressivos, apesar de acrescentarem "anos à vida", não acrescentam "vida aos anos"11.

O câncer de mama atualmente não é um vaticínio de morte; tende a ser uma doença crônica, e não mais fatal. É necessário, portanto, saber como será a vida dessas mulheres após o tratamento ${ }^{12-15}$. Na última década, o impacto psicossocial do câncer tornou-se um aspecto central tanto no que tange aos cuidados com a doença quanto às pesquisas sobre ela. Mais e mais pesquisas têm se focado em aspectos específicos da qualidade de vida, outrora negligenciados, como imagem corporal e sexualidade ${ }^{16-18}$. Ainda assim, há poucos dados referentes a esse período, que compreende o fim do tratamento primário e a sobrevida ${ }^{17}$. Várias pesquisas sugerem que problemas de funcionamento sexual são comuns ${ }^{12,13,17,19}$, mas há também declínio da qualidade de vida, imagem corporal, humor e relações familiares ${ }^{13,20}$.

O presente estudo visou a conhecer aspectos da qualidade de vida, inclusive a sexual, de mulheres residentes em uma cidade do interior de São Paulo após o advento do câncer de mama e a avaliar a associação entre qualidade de vida e características sociodemográficas. Foram avaliadas mulheres submetidas à cirurgia conservadora (quadrantectomia) e radical (mastectomia com e sem reconstrução imediata), com o intuito de se conhecerem possíveis diferenças na qualidade de vida desses grupos.

\section{Métodos}

\section{Tamanho amostral}

A qualidade de vida foi avaliada com o uso da técnica multivariada de análise fatorial. Segundo $\mathrm{Hair}^{21}$, são necessárias cinco replicações (no caso, mulheres) para cada variável a ser considerada no estudo, sendo que o menor tamanho amostral deverá ser de 100 replicações. O WHOQOL-bref, ao ser validado, foi agrupado em quatro domínios, e há 18 questões específicas para mulheres que tiveram câncer de mama, sendo que cada questão que avalie a qualidade de vida é considerada uma variável ${ }^{18,22}$. Somando-se os quatro domínios (quatro variáveis) a essas 18 questões, temos 22 variáveis e, segundo o critério acima, o tamanho amostral foi de 110 pacientes.

\section{Sujeitos}

Foi feito um estudo observacional analítico de corte transversal. As participantes do estudo foram selecionadas no ambulatório de Oncologia Mamária do Centro de Atenção Integral à Saúde da Mulher (CAISM) da Universidade Estadual de Campinas (UNICAMP). Cento e doze mulheres foram convidadas a participar do estudo e apenas duas se recusaram, sendo a taxa de participação de $98,2 \%$. As duas pacientes que se recusaram alegaram falta de tempo para a entrevista. Todas as mulheres assinaram um termo de consentimento livre e esclarecido. Este trabalho foi aprovado pelo Comitê de Ética em Pesquisa da Faculdade de Ciências Médicas (FCM) da UNICAMP.

Os critérios de inclusão foram: ser sobrevivente de câncer de mama (diagnóstico histopatológico de carcinoma 
da mama) e ter completado o tratamento há pelo menos um ano. Os critérios de exclusão foram: antecedente pessoal de outro(s) câncer(es) além do de mama, estar em vigência de tratamento quimioterápico ou radioterápico para o câncer de mama e ter apresentado recidiva da doença.

Foram aplicados questionários para a obtenção de dados relativos ao status socioeconômico, utilizando-se os critérios da Associação Brasileira de Anunciantes (ABA) e da Associação Brasileira dos Institutos de Pesquisa de Mercado (ABIPEME) ${ }^{23}$.

A média de idade das entrevistadas foi de 56,4 anos e o tempo desde o diagnóstico variou de 1,1 a 25,8 anos, com mediana de $7,7 \pm 4,8$ anos. Dentre as participantes, $83,6 \%$ eram brancas e $68,2 \%$ tinham companheiro fixo, sendo que $30 \%$ do total não estavam tendo relações sexuais. Quanto à escolaridade, a maioria das participantes tinha cursado até o nível fundamental I (60,4\%). Em 52,7\% dos casos, o chefe de família era outra pessoa que não a entrevistada, e mais de $80 \%$ delas pertenciam às classes sociais C, D e E. Quanto ao tipo de cirurgia, 43,6\% haviam sido submetidas à mastectomia radical (MR), 41,8\% à quadrantectomia com linfadenectomia axilar (QUAX) e $14,6 \%$ à mastectomia radical com reconstrução imediata (MRRI). A média de idade das mulheres tratadas com MR (58,7 anos) foi significativamente maior do que as submetidas à QUAX/MRRI (54,6 anos) ( $\mathrm{p}=0,03)$.

\section{Avaliação da qualidade de vida e da sexualidade}

Foi utilizado um questionário da OMS (Organização Mundial de Saúde) sobre qualidade de vida, o WHOQOLbref, uma versão abreviada do WHOQOL-100, e validado em português. Foram elaboradas questões que abordavam a sexualidade em relação à percepção da mulher quanto à sua sexualidade; abordou-se também a forma como a mulher acredita que outras pessoas a veem como ser sexual. Seis questões referentes à sexualidade formaram dois grupos de três questões, denominados componente ou fator intrínseco (intimidade) e componente ou fator extrínseco (atratividade). As questões referentes ao fator intrínseco são: percepção da mulher sobre melhora ou piora da vida sexual após a cirurgia, desejo da mulher em fazer sexo após a cirurgia e impressão da mulher sobre si mesma, como pessoa sexualmente atraente, após a cirurgia. As questões referentes ao fator extrínseco são: a impressão que a mulher tem de sua aparência após a cirurgia, o desconforto ou não em usar decotes ou ir à praia/piscina e, por último, a percepção em relação ao desejo do parceiro por ela após a cirurgia. Os questionários foram administrados pela pesquisadora em forma de entrevista.

\section{Análise estatística}

As variáveis foram estudadas quanto à resposta com o emprego das frequências absolutas e relativas. Para avaliar a consistência interna e validade dos questionários aplicados foi utilizado o coeficiente alpha de Cronbach. A análise fatorial foi utilizada para se determinarem os domínios que avaliassem a sexualidade das mulheres, por meio dos questionários aplicados, e a validade interna das questões de cada domínio resultante foi avaliada por meio do coeficiente alpha de Cronbach. Utilizaram-se como critérios para análise fatorial a rotação máxima da variância (rotation varmax) e o critério do autovalor (eigenvalue criteria). $\mathrm{O}$ valor de alpha para as questões de sexualidade foi de 0,72 .

As variáveis sociodemográficas foram avaliadas nos domínios do questionário da OMS e nos domínios de sexualidade (resultantes da análise fatorial) através do teste de Kruskal-Wallis seguido de Mann-Whitney. Para se compararem os domínios de sexualidade entre os grupos submetidos a diferentes cirurgias com ajuste para idade, transformaram-se matematicamente os fatores envolvidos para obter distribuição normal; então, aplicou-se a análise de variância (ANOVA) em dois fatores. Foi utilizado o índice de correlação de Spearman para avaliar a força da relação entre as variáveis. O nível de significância assumido foi de $5 \%$. Utilizou-se o software SAS versão 8.2.

\section{Resultados}

Em relação aos escores de qualidade de vida avaliados segundo os domínios da OMS, não houve diferença significativa nos domínios físico, de meio ambiente, psicológico ou de relações sociais quando analisados por faixa etária, nível de escolaridade, tipo de cirurgia e tempo decorrido desde a cirurgia. Mulheres com relacionamentos estáveis tiveram escores médios melhores que as sem relacionamento tanto no domínio psíquico $(p=0,04)$ como de relações sociais $(p=0,02)$. Em relação ao nível socioeconômico, tanto mulheres das classes A e $\mathrm{B}$ quanto as da classe $\mathrm{C}$ tiveram melhores escores do que as de classe $\mathrm{D}$ e $\mathrm{E}$ nos domínios físico $(\mathrm{p}=0,01)$ e meio ambiente $(\mathrm{p}=0,002)$ (Tabela 1$)$.

Em relação aos domínios relacionados à sexualidade, houve diferença significativa relacionada ao componente extrínseco ( $\mathrm{p}=0,358)$, sendo que mulheres de 30 a 39 anos tiveram escores médios piores do que as de 40 a 49 anos ( $\mathrm{p}=0,046)$ e do que as de 60 a 69 anos $(\mathrm{p}=0,04)$. Mulheres de 50 a 59 anos também tiveram escores piores do que mulheres de 60 a 69 anos $(p=0,02)$. Não houve diferença no componente intrínseco. Mulheres com relacionamentos estáveis tiveram escores melhores do que as sem relacionamento tanto no componente intrínseco $(\mathrm{p}=0,004)$ como no extrínseco $(\mathrm{p}=0,03)$. A escolaridade também afetou a sexualidade, sendo que as mulheres que cursaram até o ensino fundamental tiveram escores menores do que aquelas de nível universitário $(\mathrm{p}=0,01)$ 
nas questões referentes ao fator intrínseco. Essa diferença foi significativa apenas para esses dois grupos, sendo que entre analfabetas, mulheres com ensino médio e universitárias não houve diferença (Tabela 2).

Em relação a como as mulheres acreditam que os outros as veem sexualmente (componente extrínseco), aquelas submetidas à QUAX ou à MRRI apresentaram melhores escores quando comparadas às mastectomizadas sem reconstrução. O tempo decorrido desde a cirurgia não influenciou nos aspectos de sexualidade estudados (Tabela 2).

Apenas 17 das entrevistadas $(15,5 \%)$ estavam fazendo uso de tamoxifeno no momento da entrevista e apresentaram escores significativamente inferiores no domínio de meio ambiente $(\mathrm{p}=0,02)$ em comparação às não-usuárias. O tempo de uso não foi significativo para nenhum dos domínios de qualidade de vida e de sexualidade (resultados não apresentados em tabela).
O índice de correlação de Spearman para as questões de sexualidade demonstrou associação positiva e significativa entre todas as variáveis, exceto quando correlaciona o desejo sexual da mulher à sensação de conforto desta ao ir à praia ou usar decotes (Tabela 3). A mesma correlação foi observada para os domínios psicológico, relações sociais e meio ambiente em relação ao fator intrínseco, não tendo sido significativa para o domínio físico. Quando correlacionados os domínios do questionário da OMS com o fator extrínseco, foi significativo apenas para o domínio psicológico (Tabela 3).

Uma das questões agrupadas pela análise como pertencente ao componente extrínseco foi também analisada separadamente, pois pode referir-se a um conceito denominado fobia social: "sinto-me desconfortável se tenho que ir à praia ou usar roupas com decotes". Observou-se que mulheres submetidas à MR sem reconstrução sentem-

Tabela 1 - Média (x), desvio padrão (dp) e mediana (M) dos escores de qualidade de vida segundo os domínios da OMS de acordo com características sociodemográficas, tipo e tempo de cirurgia

\begin{tabular}{|c|c|c|c|c|c|c|c|c|c|c|c|c|c|}
\hline & \multirow{2}{*}{ n } & \multicolumn{3}{|c|}{ Físico } & \multicolumn{3}{|c|}{ Meio ambiente } & \multicolumn{3}{|c|}{ Psicológico } & \multicolumn{3}{|c|}{ Relações sociais } \\
\hline & & $x$ & $d p$ & $M$ & $x$ & $d p$ & M & $x$ & $d p$ & $M$ & $\mathrm{x}$ & $d p$ & $M$ \\
\hline \multicolumn{14}{|l|}{ Idade (anos) } \\
\hline $30-39$ & 6 & 86,3 & 13,4 & 91,1 & 61,5 & 7,3 & 60,9 & 54,9 & 10,3 & 60,4 & 70,8 & 15,6 & 70,8 \\
\hline $40-49$ & 20 & 78,6 & 13,2 & 78,6 & 53,4 & 11,2 & 54,7 & 58,3 & 12,7 & 62,5 & 71,3 & 19,2 & 75 \\
\hline $50-59$ & 44 & 78,6 & 15,6 & 82,1 & 57,6 & 9 & 56,3 & 60,1 & 11 & 60,4 & 66,9 & 14,4 & 66,7 \\
\hline $60-69$ & 31 & 74,8 & 14,1 & 75 & 56,6 & 9,7 & 56,3 & 59,4 & 10,6 & 62,5 & 70,2 & 14,1 & 75 \\
\hline \multicolumn{14}{|l|}{ Estado marital* } \\
\hline Casada/estável & 75 & 77,7 & 14,1 & 82,1 & 56,4 & 9,5 & 56,3 & 61,4 & 9,4 & 62,5 & 71,9 & 12 & 75 \\
\hline Sem companheiro & 35 & 76,5 & 15,2 & 78,6 & 56,8 & 10,3 & 56,3 & 55,7 & 12,9 & 58,3 & 63,8 & 18,6 & 66,7 \\
\hline Valor de $\mathrm{p}$ & & \multicolumn{3}{|c|}{0,6832} & \multicolumn{3}{|c|}{0,8166} & \multicolumn{3}{|c|}{0,0438} & \multicolumn{3}{|c|}{0,0182} \\
\hline \multicolumn{14}{|l|}{ Escolaridade } \\
\hline Superior & 9 & 79 & 18,3 & 89,3 & 61,1 & 9,9 & 62,5 & 61,1 & 14,1 & 66,7 & 74,1 & 18,4 & 75 \\
\hline Valor de p & & \multicolumn{3}{|c|}{0,1914} & \multicolumn{3}{|c|}{0,4058} & \multicolumn{3}{|c|}{0,8651} & \multicolumn{3}{|c|}{0,4237} \\
\hline \multicolumn{14}{|l|}{ Nível socioeconômico } \\
\hline A/B & 14 & 81,6 & 12,4 & 85,7 & 61 & 9,4 & 60,9 & 61,3 & 13,9 & 64,9 & 75 & 11,8 & 75 \\
\hline C & 51 & 80 & 14,5 & 82,1 & 59 & 8,1 & 59,4 & 60,7 & 10,6 & 62,5 & 71,4 & 13,8 & 75 \\
\hline D/E & 45 & 72,9 & 14 & 75 & 52,4 & 10,1 & 53,1 & 57,9 & 10,2 & 58,3 & 65,2 & 16 & 66,7 \\
\hline Valor de $p$ & & \multicolumn{3}{|c|}{$0,0135 \#$} & \multicolumn{3}{|c|}{$0,0021 \#$} & \multicolumn{3}{|c|}{0,1101} & \multicolumn{3}{|c|}{0,1269} \\
\hline \multicolumn{14}{|l|}{ Tipo cirurgia* } \\
\hline Mastectomia & 48 & 75,7 & 14 & 78,6 & 56 & 10,1 & 56,3 & 58,9 & 10,7 & 58,3 & 67 & 15,8 & 66,7 \\
\hline MRRI / QUAX & 62 & 78,6 & 14,6 & 82,1 & 57 & 9,4 & 56,3 & 60,1 & 11,1 & 62,5 & 71,1 & 13,9 & 75 \\
\hline
\end{tabular}

teste de Kruskal Wallis; MRRI: mastectomia radical com reconstrução imediata; *teste de Wilcoxon; QUAX: Quadrantectomia com linfadenectomia axilar; \#A/B $\neq$ $D / E ; C \neq D / E$. 
Tabela 2 - Média (x), desvio padrão (dp) e mediana (M) dos escores de sexualidade segundo características sociodemográficas, tipo e tempo de cirurgia

\begin{tabular}{|c|c|c|c|c|c|c|c|}
\hline & \multirow{2}{*}{ n } & \multicolumn{3}{|c|}{ Fator intrínseco } & \multicolumn{3}{|c|}{ Fator extrínseco } \\
\hline & & $x$ & $d p$ & M & $x$ & $d p$ & M \\
\hline \multicolumn{8}{|l|}{ Idade (anos) } \\
\hline $30-39$ & 6 & 53,1 & 29,8 & 46,5 & 32 & 20,4 & 30,7 \\
\hline $40-49$ & 20 & 54,9 & 22,8 & 53,1 & 53,4 & 18,5 & 50,9 \\
\hline $50-59$ & 44 & 49,7 & 18,5 & 54 & 44,5 & 19,4 & 40 \\
\hline $60-69$ & 31 & 52,1 & 19,5 & 57,2 & 55 & 21,7 & 58,9 \\
\hline 70 ou + & 9 & 63,7 & 19,7 & 63,4 & 52,3 & 18 & 56,7 \\
\hline Valor de $p$ & & \multicolumn{3}{|c|}{0,4691} & \multicolumn{3}{|c|}{$0,0358^{\star \star}$} \\
\hline \multicolumn{8}{|l|}{ Estado marital* } \\
\hline Casada/estável & 75 & 55,7 & 20,5 & 61 & 52 & 20,6 & 53,2 \\
\hline Sem companheiro & 35 & 45,5 & 17 & 49,9 & 42,4 & 18,9 & 38,8 \\
\hline Valor de $\mathrm{p}$ & & \multicolumn{3}{|c|}{0,0036} & \multicolumn{3}{|c|}{0,0280} \\
\hline \multicolumn{8}{|l|}{ Escolaridade } \\
\hline Analfabeta & 8 & 61,4 & 14 & 61,4 & 39 & 26,1 & 32 \\
\hline Fundamental & 75 & 48,8 & 19,4 & 53 & 51,1 & 20,5 & 53,4 \\
\hline Médio & 14 & 58,6 & 23,4 & $57,4^{*}$ & 44,5 & 18,7 & 49,4 \\
\hline Superior & 9 & 67,5 & 16,2 & $70,8^{\star}$ & 52,1 & 13,7 & 51,6 \\
\hline Valor de p & & \multicolumn{3}{|c|}{$0,0111^{\#}$} & \multicolumn{3}{|c|}{0,3890} \\
\hline \multicolumn{8}{|l|}{ Nível socioeconômico } \\
\hline A/B & 14 & 62,5 & 17 & 68,3 & 54 & 18,6 & 52,4 \\
\hline C & 51 & 51,3 & 21,2 & 51,5 & 48,3 & 19,5 & 51,3 \\
\hline D/E & 45 & 50,9 & 18,9 & 55 & 48,2 & 22,3 & 49,2 \\
\hline Valor de p & & \multicolumn{3}{|c|}{0,0673} & \multicolumn{3}{|c|}{0,6856} \\
\hline \multicolumn{8}{|l|}{ Tipo de cirurgia ${ }^{8}$} \\
\hline Mastectomia & 48 & 49,9 & 16,2 & 52,9 & 43,7 & 20,3 & 38,8 \\
\hline MRRI /QUAX & 62 & 54,6 & 22,4 & 59,3 & 53 & 19,9 & 53,1 \\
\hline Valor de p & & \multicolumn{3}{|c|}{0,3372} & \multicolumn{3}{|c|}{0,0158} \\
\hline \multicolumn{8}{|l|}{$\begin{array}{l}\text { Tempo desde a cirurgia } \\
\text { (anos) }\end{array}$} \\
\hline Até 5 & 36 & 51,4 & 22 & 55 & 50 & 19,9 & 48,5 \\
\hline 6 a 10 & 44 & 52,2 & 20,2 & 57,7 & 44,5 & 21,7 & 38,8 \\
\hline Mais de 10 & 30 & 54,4 & 17,5 & 54,7 & 54,3 & 18,5 & 55,3 \\
\hline Valor de $\mathrm{p}$ & & \multicolumn{3}{|c|}{0,8709} & \multicolumn{3}{|c|}{0,1685} \\
\hline
\end{tabular}

teste de Kruskal-Wallis; * teste de Wilcoxon; \& ANOVA; \# ensino fundamental; \# ensino superior; MRRI: mastectomia radical com reconstrução imediata; QUAX: quadrantectomia com linfadenectomia axilar; **30-39 versus $40-49$ $(p=0,046) ; 30-39$ versus $60-69(p=0,04) ; 50-59$ versus $60-69(p=0,02)$. se mais à vontade para usar roupas com decotes do que as mulheres submetidas à QUAX ou à MRRI $(\mathrm{p}<0,01)$ (Tabela 4).

\section{Discussão}

Os melhores escores foram obtidos no domínio físico e os piores, no domínio psicológico, porém sempre superiores a 55, confirmando uma boa qualidade de vida dessas mulheres ${ }^{24}$. Os resultados são compatíveis com outro estudo na mesma instituição, que comparou a qualidade de vida em mulheres com e sem câncer de mama. Uma hipótese para a melhor qualidade de vida tanto nos aspectos físicos como nos psicológicos seria reflexo da assistência multiprofissional oferecida ${ }^{24}$.

Em mulheres norte-americanas com história de câncer de mama também se observou boa qualidade de vida. Em um estudo sobre qualidade de vida em mulheres alemãs com câncer de mama foram observados escores similares aos da população geral ${ }^{25}$. A importância do apoio à mulher com câncer de mama pode ser observada neste estudo, pois mulheres com companheiro apresentaram escores significativamente melhores nos domínios psicológico e relações sociais. Já mulheres sem companheiro e sem a mama, ou com deformidade pós-cirúrgica da mama, podem sentir-se inferiorizadas se comparadas àquelas que possuem mamas não operadas e, por isso, menos capazes de estabelecer novos vínculos afetivos. Mulheres sem companheiro parecem ser mais vulneráveis a problemas de ajustamento, sobretudo no campo dos relacionamentos ${ }^{26}$.

Também se observou que mulheres de estrato social mais elevado têm melhores escores nos domínios físico e meio ambiente. Tanto a presença de um companheiro quanto o melhor extrato social podem ser indicativos de que essas mulheres têm mais chances de obter apoio social e psíquico e têm melhores condições de moradia.

Tabela 3 - Correlação entre os domínios de qualidade de vida e sexualidade segundo correlação de Spearman - coeficientes de Spearman (valor de p)

\begin{tabular}{|c|c|c|c|c|c|c|}
\hline Domínios & Físico & Psicológico & Relações sociais & Meio ambiente & Fator intrínseco & Fator extrínseco \\
\hline Físico & 1,0 & $0,4(p<0,0001)$ & $0,43(p<0,0001)$ & $0,27 \quad(p=0,0039)$ & $0,176(p=0,06)$ & $-0,04(p=0,6)$ \\
\hline Relações sociais & & & 1,0 & $0,31 \quad(p=0,0008)$ & $0,31136(p=0,001)$ & $0,1 \quad(\mathrm{p}=0,2551)$ \\
\hline Fator intrínseco & & & & & 1,0000 & $0,08(p=0,3)$ \\
\hline Fator extrínseco & & & & & & 1,0 \\
\hline
\end{tabular}

alpha de Cronbach $=0,67$.

Tabela 4 - Correlação entre as questões referentes aos domínios de sexualidade segundo correlação de Spearman - coeficientes de Spearman (valor de p)

\begin{tabular}{|c|c|c|c|c|c|c|}
\hline Questões & Aparência & Ir à praia & Desejo do parceiro & Desejo da mulher & Atruente & Sexo \\
\hline Aparência & 1,0000 & $0,36639(p<0,0001)$ & $0,20399(\mathrm{p}=0,0326)$ & $0,23173(p=0,0149)$ & $0,35991 \quad(p=0,0001)$ & $0,24438(p=0,0101)$ \\
\hline Desejo do parceiro & & & 1,0000 & $0,24021 \quad(p=0,0115)$ & $0,31134(p=0,0009)$ & $0,42867(p<0,0001)$ \\
\hline Atruente & & & & & 1,0000 & $0,36171(p=0,0001)$ \\
\hline Sexo & & & & & & 1,0000 \\
\hline
\end{tabular}


Não houve diferença significativa entre mulheres submetidas à QUAX ou MRRI e mastectomizadas sem reconstrução, exceto no componente extrínseco. Mulheres submetidas à MR tiveram escores mais baixos, ainda que todas apresentassem escores baixos. Isso é compatível com muitos estudos em que mulheres quadrantectomizadas tinham qualidade de vida significativamente melhor que mastectomizadas com ou sem reconstrução $0^{5,6,27}$. Outros trabalhos, no entanto, mostraram declínio do funcionamento sexual em mulheres com MRRI em relação às QUAX ou MR. Isso pode ser, em parte, explicado pela alteração tátil que ocorre com a prótese ou com os retalhos miocutâneos, pela ausência de mamilo e pela assimetria usualmente observada entre a mama normal e a reconstruída ${ }^{3}$.

Em nosso estudo, mulheres mais jovens apresentaram escores piores em relação à sexualidade no que diz respeito ao fator extrínseco ou atratividade (como elas acreditam que os outros as veem). Isso é compatível com dados da literatura, sendo que a maioria das pesquisas indica que mulheres mais jovens têm qualidade de vida inferior após o diagnóstico de câncer de mama ${ }^{28,29}$. Elas têm maior risco para estresse psicológico e para disfunção sexual, quando comparadas às mulheres mais velhas ${ }^{30}$. Mulheres mais velhas costumam ter mais resiliência, aceitando melhor a doença e seu tratamento. Já as mais jovens, além de terem menos preparo para trabalhar a questão, podem ter também planos de maternidade adiados ou mesmo abandonados em consequência do tratamento, que pode levar à menopausa precoce ${ }^{30}$. Isso talvez explique o fato de termos encontrado em nosso estudo maior desconforto por parte das mulheres com QUAX ou MRRI em usar decotes ou em ir à praia quando comparadas às mulheres com MR, que foram significativamente mais idosas quando comparadas ao primeiro grupo.

Mulheres com companheiro fixo apresentaram escores melhores que as sem companheiro tanto no componente intrínseco quanto no extrínseco. $\mathrm{O}$ apoio dos companheiros parece ter um peso importante na sexualidade da mulher. $\mathrm{O}$ advento do câncer de mama pode fortalecer alguns relacionamentos e ter efeitos negativos em outros, muitas vezes pela dificuldade em se discutirem sentimentos ou mesmo pela percepção da mulher de perda da feminilidade ${ }^{31}$. Em demais estudos, mulheres com companheiro fixo obtiveram melhores escores de qualidade de vida ${ }^{13,30}$. No entanto, em estudo precedente nessa instituição, observou-se uma associação negativa entre mulheres casadas e qualidade de vida. Isso porque a qualidade do relacionamento deve influenciar diretamente a qualidade de vida. Talvez as mulheres deste estudo, como os próprios autores salientam, possuíssem relacionamentos pouco satisfatórios ${ }^{24}$.

Mulheres com taxas mais altas de escolaridade tiveram melhor autopercepção da sexualidade. Em um estudo com mulheres norte-americanas, aquelas com maior escolaridade também tiveram melhores taxas de qualidade de vida ${ }^{30}$. Isso pode estar relacionado ao fato de apresentarem mais recursos internos para lidar com a doença, maior apoio e compreensão familiar e uma noção mais aproximada da extensão da enfermidade. Os achados deste estudo não condizem com outros da literatura, que surpreendentemente encontraram pior funcionamento sexual nos domínios de interesse e satisfação em mulheres com maior escolaridade ${ }^{26}$. Uma hipótese para esses resultados foi a do maior nível de estresse ocupacional verificado em mulheres com maior escolaridade quando comparadas às donas de casa e/ou trabalhadoras braçais.

Todas as questões se relacionaram significativamente, exceto desejo da mulher de ir à praia. Essas questões são delicadas e, sem dúvida, interferem na qualidade de vida das mulheres e merecem aprofundamento futuro com estudos qualitativos. Essa atenção pode contribuir para uma melhor percepção de saúde, minimizando o impacto do tratamento do câncer de mama na qualidade de vida. Estudos prévios demonstraram a importância do suporte psicossocial às pacientes com câncer de mama ${ }^{32-34}$.

Os resultados obtidos na avaliação segundo os domínios da OMS associados aos obtidos nos domínios de sexualidade sugerem multifatoriedade na QDV. Especificamente em relação à sexualidade, exercê-la de forma plena e sentirse atraente é mais importante que sentir-se bem ao usar decotes, que o desejo sexual do parceiro e mesmo, que a sua própria aparência. Conclui-se que a sexualidade é uma questão importante na qualidade de vida. Para a grande maioria das mulheres, o desejo de alcançar a intimidade é a força motriz do ciclo de resposta sexual da mulher, que começa na necessidade básica de intimidade, inclui mutualismo, respeito e comunicação. Se há envolvimento emocional positivo e interação física, a intimidade é alcançada e o ciclo se fortalece ${ }^{35}$.

\section{Referências}

1. Jemal A, Siegel R, Ward E, Hao Y, Xu J, Murray T, et al. Cancer statistics, 2008. CA Cancer J Clin. 2008;58(2):71-96.

2. Ministério da Saúde. Instituto Nacional do Câncer. Estimativa de câncer: incidência de câncer no Brasil [documento da Internet]. 2008 [citado 06 fev 2009]. Disponível em: http://www.inca.gov. br/estimativa/2008/index.asp?link=conteudo_view. asp\&ID =5
3. Yurek D, Farrar W, Andersen BL. Breast cancer surgery: comparing surgical groups and determining individual differences in postoperative sexuality body change stress. J Consult Clin Psychol. 2000;68(4):697-709.

4. Jahkola T. Self-perceptions of women after early breast cancer surgery. Eur J Surg Oncol. 1998;24(1):9-14. 
5. Al-Ghazal SK, Fallowfield L, Blamey RW. Comparison of psychological aspects and patient satisfaction following breast conserving surgery, simple mastectomy and breast reconstruction. Eur J Cancer. 2000;36(15):1938-43

6. Amichetti $M$, Caffo $O$. Quality of life in patients with early stage breast carcinoma treated with conservation surgery and radiotherapy. An Italian monoinstitutional study. Tumori. 2001;87(2):78-84.

7. Anllo LM. Sexual life after breast cancer. J Sex Marital Ther. 2000;26(3):241-8.

8. Matthews BA, Baker F, Hann DM, Denniston M, Smith TG. Health status and life satisfaction among breast cancer survivor peer support volunteers. Psychooncology. 2002;1 113):199-211.

9. Ganz PA, Rowland JH, Desmond K, Meyerowitz BE, Wyatt GE. Life after breast cancer: understanding women's healthrelated quality of life and sexual functioning. J Clin Oncol. 1998; 16(2):501-14.

10. Bullinger $M$, Anderson R, Cella D, Aaronson N. Developing and evaluating cross-cultural instruments from minimum requirements to optimal models. Qual Life Res. 1993;2(6):451-9.

11. Bech P. Quality of life measurements for patients taking which drugs? The clinical PCASEE perspective. Pharmacoeconomics. 1995;7(2):141-51.

12. Broeckel JA, Thors CL, Jacobsen PB, Small M, Cox CE. Sexual functioning in long-term breast cancer survivors treated with adjuvant chemotherapy. Breast Cancer Res Treat. 2002;75(3):241-8.

13. Spagnola S, Zabora J, Brintzenhofeszoc K, Hooker C, Cohen G, Baker $F$. The satisfaction with life domains scale for breast cancer (SLDS-BC). Breast J. 2003;9(6):463-71.

14. Fialka-Moser V, Crevenna R, Korpan M, Quittan M. Cancer rehabilitation: particularly with aspects on physical impairments. J Rehabil Med. 2003;35(4):153-62.

15. Engel J, Kerr J, Schlesinger-Raab A, Sauer H, Holzel D. Quality of life following breast-conserving therapy or mastectomy: results of a 5-year prospective study. Breast J. 2004;10(3):223-31.

16. Stead ML. Sexual dysfunction after treatment for gynaecologic and breast malignancies. Curr Opin Obstet Gynecol. 2003; 15(1):57. 61.

17. Sheppard LA, Ely S. Breast cancer and sexuality. Breast J. 2008;14(2):176-81.

18. Hormes JM, Lytle LA, Gross CR, Ahmed RL, Troxel AB, Schmitz $\mathrm{KH}$. The body image and relationships scale: development and validation of a measure of body image in female breast cancer survivors. J Clin Oncol. 2008;26(8):1269-74.

19. Beckjord E, Campas BE. Sexual quality of life in women with newly diagnosed breast cancer. J Psychosoc Oncol. 2007;25(2): 19-36.
20. Ganz PA, Kwan L, Stanton AL, Krupnick JL, Rowland JH, Meyerowitz $\mathrm{BE}$, et al. Quality of life at the end of primary treatment of breast cancer: first results from the moving beyond cancer randomized trial. J Natl Cancer Inst. 2004;96(5):376-87.

21. Hair JFJ, Anderson RE, Tatham RL, Black WC. Multivariate data analysis. 5th ed. Upper Saddle River: Prentice Hall, 1998.

22. Fleck MPA, Louzada S, Xavier M, Chachamovich E, Vieira G, Santos $L$, et al. Aplicação da versão em português do instrumento abreviado de avaliação da qualidade de vida "WHOQOL-bref". Rev Saúde Pública. 2000;34(2):178-83.

23. Almeida PM, Wickerhauser $H$. O critério ABA/ABIPEME: em busca de uma atualização. São Paulo: ABA/ABIPEME, 1991.

24. Conde DM, Pinto-Neto AM, Cabello C, Santos-Sá D, Costa-Paiva L, Martinez EZ. Quality of life in Brazilian breast cancer survivors age 45-65: associated factors. Breast J. 2005; $11(6): 425-32$.

25. Arndt V, Merx H, Sturmer T, Stegmaier C, Ziegler H, Brenner $\mathrm{H}$. Age-specific detriments to quality of life among breast cancer patients one year after diagnosis. Eur J Cancer. 2004;40(5):673-80.

26. Greendale GA, Petersen L, Zibecchi L, Ganz PA. Factors related to sexual function in postmenopausal women with a history of breast cancer. Menopause. 2001;8(2):111-9.

27. Wapnir IL, Cody RP, Greco RS. Subtle differences in quality of life after breast cancer surgery. Ann Surg Oncol. 1999;6(4):359-66.

28. Mor V, Malin M, Allen S. Age differences in the psychosocial problems encountered by breast cancer patients. J Natl Cancer Inst Monogr. 1994;(16):191-7.

29. Northouse LL. Breast cancer in younger women: effects on interpersonal and family relations. J Natl Cancer Inst Monogr. 1994;(16): 183-90.

30. Ganz PA, Greendale GA, Petersen L, Kahn B, Bower JE. Breast cancer in younger women: reproductive and late health effects of treatment. J Clin Oncol. 2003;21 (22):4184-93.

31. Holmberg SK, Scott LL, Alexy W, Fife BL. Relationship issues of women with breast cancer. Cancer Nurs. 2001;24(1):53-60.

32. Fukui $S$, Kugaya A, Okamura $H$, Kamiya $M$, Koike M, Nakanishi $T$, et al. A psychosocial group intervention for Japanese women with primary breast carcinoma. Cancer. 2000;89(5):1026-36.

33. Okamura H, Fukui S, Nagasaka Y, Koike M, Uchitomi Y. Psychoeducational intervention for patients with primary breast cancer and patient satisfaction with information: an exploratory analysis. Breast Cancer Res Treat. 2003;80(3):331-8.

34. Basson R, Berman J, Burnett A, Derogatis L, Fergunson D, Fourcroy J, et al. A report of the international consensus development conference on female sexual dysfunction: definitions and classifications. J Urol. 2000; 163(3):888-93.

35. Bachmann GA. Menopausal sexuality. In: Lobo RA. Menopause: biology and pathobiology. San Diego: Academic Press; 2000. p. 383-93. 